\title{
ATOMIC DATA FOR PN ANALYSIS
}

\author{
P.J. STOREY \\ Dept. Physics \& Astronomy, University College London, \\ Gower Street, LONDON WC1E 6BT, UK
}

\section{Introduction}

This paper will review a selection of the work that has been published on the calculation of atomic parameters, relevant to $\mathrm{PN}$, in the period since the 1992 Symposium. It will also attempt to address the accuracy of theoretical results, where possible. The review will concentrate primarily on results obtained using the powerful electron-scattering codes that describe resonance phenomena with ever increasing precision. Calculations of oscillator strengths and photoionisation cross-sections will not be discussed.

\section{Electron collisional excitation}

The methods used in the calculation of cross-sections for electron collisional excitation will not be discussed in any detail here. Unless otherwise stated, the calculations described below employ a coupled-channel description of the scattering process and therefore automatically include resonance phenomena, although the accuracy of the description of resonances depends on the detail of a particular calculation.

\subsection{ATOMIC FINE-STRUCTURE TRANSITIONS}

The IRON Project is an international collaboration of computational atomic physicists who have been systematically studying the electron excitation of ground term fine-structure transitions in atomic ions. Most of the transitions lie in the infrared. The calculations have been carried out along isoelectronic sequences with data now published for most ions of the abundant elements with atomic number less than twenty. Thermally averaged collision strengths are given as a function of electron temperature. The theoretical and computational methods used by the IRON Project are described by Hummer et al (1993). The available data are given below, by sequence and by paper number in the IRON Project (IP) series; 
H-seq: He II and Fe XXVI. Results for $n \leq 4$ for He II (IP XV). B-seq: $\mathrm{Ne}$ VI, Mg VII, Al IX, Si X, P XII, Ar XIV, Ca XVI, Fe XXII (IP III). Results for earlier members of this sequence were published by Blum \& Pradhan (1992), while calculations of relative line emissivities are presented by Peng \& Pradhan (1995).

C-seq: N II to S XI inclusive (IP II). By comparing the relative intensities of optical and infrared lines of Ne V, Oliva, Pasquali \& Reconditi (1996) have concluded that the effective collision strengths given in IP II are too large by a factor of about three. They suggest that the discrepancy is caused by near threshold resonances being incorrectly positioned in the calculations, given that there is known to be a particularly large resonance enhancement in Clike neon. The positions of the near threshold resonances in this particular case were, however, discussed in great detail by Lennon \& Burke (1991) and they concluded that the calculated positions were not significantly in error. It seems more probable therefore that the problem lies in the measured $\mathrm{Ne} \mathrm{V}$ line intensities.

O-seq: $\mathrm{F}$ II to Ar XI inclusive (IP V).

F-seq: Ne II to Ca XII inclusive and Fe XVIII (IP IV).

Al-seq: Ar VI, K VII, Ca VIII, (IP XI) Fe XIV (IP XIV).

Si-seq: S III to Ca VII inclusive (IP X).

S-seq: Ar III to Ca V inclusive (IP X).

Cl-seq: Ar II to Ni XII (IP IX).

Ti-seq: V II to Ni VII inclusive (IP VIII).

V-seq: Mn III to Ni VI inclusive (IP XII).

The IRON project homepage, containing an up to date list of extant and forthcoming publications is at http://www.am.qub.ac.uk/projects/iron.

A systematic study of the convergence of calculated collision strengths and thermally averaged collision strengths was carried out by Saraph \& Storey (1996) (IP XI) for the Al-like ion Ar VI. The near threshold collision strength was found to be dominated by broad resonance features converging on the thresholds associated with the terms of the $n=3$ electron configurations. The calculated positions of these resonances were found to be sensitive to the approximations made in constructing the wave functions of the target states. As a result Saraph \& Storey (1996) found that the effective collision strength for the ground state fine-structure transition was uncertain by a factor of two for temperatures less than about $5000 \mathrm{~K}$. The uncertainty at $10000 \mathrm{~K}$ was approximately $25 \%$. Uncertainties of at least this magnitude can be expected to apply to all calculations involving $n=3$ valence electrons. 


\section{ATOMIC DATA FOR PN ANALYSIS}

\subsection{IRON IONS}

Members of the IRON Project have also carried out calculations for the ions of iron. Thermally averaged collision strengths are published for Fe I (IP XXII), Fe II (IP VI, XIII) and Fe III (IP XVIII) as described below. Fe I: Thermally averaged collision strengths have been calculated for the levels of the ground ${ }^{5} \mathrm{D}$ and first excited ${ }^{5} \mathrm{~F}$ terms, valid for temperatures up to $4000 \mathrm{~K}$. The authors estimate the uncertainties in their results to be in the region of 10 to $20 \%$. Unlike the calculations for ions described above, the collision strength for scattering from neutrals is not necessarily dominated by resonances and this is indeed the case for Fe I.

Fe II and Ni II: Thermally averaged collision strengths have been calculated among the energetically lowest 142 fine-structure levels of Fe II by Zhang \& Pradhan (1995) (IP VI). The calculations have been carried out for temperatures from 1000 to $100000 \mathrm{~K}$, but only a small subset of these are published, the remainder being available electronically from the authors. The calculation described by Bautista \& Pradhan (1996) in IP XIII gives some additional results for forbidden transitions in Fe II and also for fobidden transitions in Ni II. There are significant differences between thermally averaged collision strengths given in IP VI and IP XIII (Bautista \& Pradhan 1996) due to changes in the target and scattering wave functions, which must be viewed as a measure of the uncertainty in these calculations.

\subsection{FORBIDDEN LINES OF TRACE ELEMENTS}

Following the detection of forbidden lines of elements from rows 4,5 and 6 of the periodic table (Péquignot \& Baluteau 1994), Schöning \& Butler (1995) and Schöning (1995) have calculated thermally averaged collision strengths for the ground configuration forbidden transitions of $\mathrm{Kr}$ IV and Xe IV for temperatures in the range $2000-50000 \mathrm{~K}$. These calculations rely on an LS-coupling description of the scattering problem with a subsequent transformation to pair coupling and cannot therefore be regarded as definitive until relativistic effects have been fully examined. Nevertheless, the authors are able to rule out the very large enhancements in the collision strengths of more than a factor of ten that were proposed by Péquignot \& Baluteau (1994) to account for the strength of the observed lines.

\subsection{NEUTRAL HELIUM}

The most recent work on the excitation of helium by electrons is that of Sawey \& Berrington (1993). They had already computed collision strengths for the 29 states with $n \leq 5$ (Sawey et al, 1990) but the collision strengths for many allowed transitions were incomplete due to truncation of the par- 


\section{P.J. Storey}

tial wave expansion. The new calculation extends the partial wave expansion but results are not given for transitions where the contribution from missing partial waves still exceeds $50 \%$. This problem is particularly severe for transitions among the states with $n=4$. In keeping with earlier papers, the authors do not give data for excitation to the $n=5$ states, arguing that these will be incorrect unless at least $n=6$ states are included in the scattering problem. Effective collision strengths are given for temperatures from $2000-30000 \mathrm{~K}$ and collision strengths are compared graphically with the results of the earlier 19-state $(n \leq 4)$ calculation of Berrington $\&$ Kingston (1987). The comparisons confirm that the 19-state calculation did overestimate the collision strengths between the $n=2$ and $n=4$ states as predicted.

\subsection{OTHER IONS}

O II: Collision strengths and thermally averaged collision strengths for transitions among the terms of the $2 \mathrm{~s}^{2} 2 \mathrm{p}^{3}$ and $2 \mathrm{~s} 2 \mathrm{p}^{4}$ configurations have been calculated by McLaughlin \& Bell (1993a, 1993b). These calculations extend from threshold to a free electron energy of 30 Rydbergs and thermally averaged collision strengths are given from $5 \times 10^{3}-1 \times 10^{6} \mathrm{~K}$. The collision strengths between fine-structure levels are not given.

Mg II: Collision strengths and thermally averaged collision strengths among the energetically lowest 17 fine-structure levels have been calculated by Sigut \& Pradhan (1995). Comparison is made with experimental measurements for the 3s-3p resonance transition and excellent agreement is found within 1 Rydberg of threshold.

S II: Recent studies of electron excitation of S II show significant differences. The calculation of Ramsbottom, Bell \& Stafford (1996) shows an important resonance close to threshold in the ${ }^{4} \mathrm{~S}^{\mathbf{o}}-{ }^{2} \mathrm{P}^{\mathbf{o}}$ collision strength (see Keenan et al 1996), which is absent in the earlier calculation of Cai \& Pradhan (1993). This leads to differences of a factor of approximately two in the thermally averaged collision strengths for this transition. A resolution of this discrepancy must await a detailed analysis of resonance positions similarto that carried out for $\mathrm{Ne} \mathrm{V}$ (see section 2.1 above) by Lennon \& Burke (1991).

\section{Recombination processes}

\subsection{H-LIKE AND HELIUM RECOMBINATION}

H-like ions: New, very extensive, tables of total recombination coefficients, effective recombination coefficients for lines and departure coefficients as a function of $n$ and $n l(n \leq 50)$ have been given by Storey \& Hummer 


\section{ATOMIC DATA FOR PN ANALYSIS}

(1995) for hydrogenic ions with nuclear charge $Z=1-8$. The calculations cover the electron density range $\log N_{e}=2(1) 14$ and temperatures from 500 to $100000 \mathrm{~K}$. The paper contains only total recombination coefficients, the remainder of the data set being available by anonymous ftp from the CDS archive.

He-I: Smits (1996) has presented new theoretical emissivities for optical recombination lines of helium. This paper improves on and corrects an earlier paper by the same author (Smits, 1991) in which inaccurate recombination coefficients were used. The predicted strengths of the strongest optical recombination lines now agree with the earlier work of Brocklehurst (1972) to within $2 \%$, except for the $\lambda 7065$ transition, where Smits (1991) had already pointed out an error in the work of Brocklehurst. Electron collisional processes among the $n=2$ states are included but not collisional excitation of higher states from the $2^{3} \mathrm{~S}$ metastable. These collisional effects have been discussed by Kingdon \& Ferland (1995), who combined the work of Smits (1996) with the latest calculated collision strengths for He I by Sawey \& Berrington (1993) to compute the relative contributions to the strongest optical lines from recombination and collisional excitation.

\subsection{TOTAL RECOMBINATION COEFFICIENTS}

Elaborate calculations of total recombination coefficients have been carried out by Nahar \& Pradhan (1994), including the effects of both radiative (non-resonant) and dielectronic recombination. Nahar (1995) has tabulated total recombination coefficients for the recombined ions Si I, Si II, S II, S III, $\mathrm{C}$ II and the C-like ions C I to Si IX inclusive and S XI in the temperature range $\log T=1-9$. Recombination coefficients to individual terms are also tabulated for temperatures up to $10000 \mathrm{~K}$. These calculations rely on detailed photoionisation cross-sections, which include part of the dielectronic recombination via resonances. As discussed above in section 2.1 in relation to collision rates, this approach is vulnerable to errors in the calculated resonance positions, so, if the recombination coefficient at nebular temperatures exhibits a strong dielectronic enhancement, there may be significant uncertainties.

\subsection{RECOMBINATION LINES FROM MANY-ELECTRON IONS}

Detailed photoionisation cross-sections, derived from elaborate scattering calculations, have been used by Storey (1994) and Liu et al (1995), to study the recombination spectrum of $\mathrm{O}$ II. Their results are only applicable at nebular temperatures, $(T \leq 15000 \mathrm{~K})$ since high temperature dielectronic recombination is not fully treated, but the full effects of cascading and a partial treatment of electron collisions are included. Effective recombination 


\section{P.J. Storey}

coefficients are tabulated for all the strong lines of O II in LS-coupling by Storey (1994), while Liu et al (1995) investigate the breakdown of LScoupling that occurs for the $4 \mathrm{f}-3 \mathrm{~d}$ and $3 \mathrm{~d}-3 \mathrm{p}$ transition arrays. Excellent agreement is found between the predicted line intensities and those observed in deep optical spectra of NGC 7009.

\section{References}

Berrington, K.A. \& Kingston, A.E. 1987, J. Phys. B: At. Mol. Opt. Phys. 20, 6631

Blum, R.D. \& Pradhan, A.K. 1992, ApJS 80, 245

Brocklehurst, M. 1972, MNRAS 153, 471

Cai, W. \& Pradhan, A.K. 1993, ApJS 88, 329

IP I Hummer, D.G., Berrington, K.A., Eissner, W., Pradhan, A.K., Saraph, H.E. \& Tully, J.A 1993, A\&A 279, 298

IP II Lennon, D.J. \& Burke, V.M. 1994, A\&AS 103, 273

IP III Zhang, H.L., Graziani, M \& Pradhan A.K. 1994, A\&A 283, 319

IP IV Saraph, H.E. \& Tully, J.A. 1994, A\&AS 107, 29

IP V Butler, K. \& Zeippen, C.J. 1994, A\&AS 108, 1

IP VI Zhang, H.L. \& Pradhan, A.K. 1995, A\&A 293, 953

IP VIII Berrington, K.A. 1995, A\&A 109, 193

IP IX Pelan, J.C. \& Berrington, K.A. 1995, A\&AS 110, 209

IP X Galavis, M.E., Mendoza, C. \& Zeippen, C.J. 1995, A\&AS 111, 347

IP XI Saraph, H.E. \& Storey, P.J. 1996, A\&AS 115, 151

IP XII Berrington, K.A. \& Pelan, J.C. 1995, A\&AS 114, 367

IP XIII Bautista, M.A. \& Pradhan, A.K. 1996, A\&AS 115, 551

IP XIV Storey, P.J., Mason, H.E. \& Saraph, H.E. 1996, A\&AS 309, 677

IP XV Kisielius, R., Berrington, K.A. \& Norrington, P.H. 1996, A\&AS 118, 157

IP XVIII Zhang, H.L. 1996, A\&AS 119, 523

IP XXI Pelan, J. \& Berrington, K.A. 1997, A\&AS in press

Keenan, F.P., Aller, L.H., Bell, K.L., Hyung, S, McKenna, F.C. \& Ramsbottom, C.A. 1996, MNRAS 281, 1073

Kingdon, J. \& Ferland, G.J. 1995, ApJ 442, 714

Lennon, D.J. \& Burke, V.M. 1991, MNRAS 251, 628

Liu, X.-W., Storey, P.J., Barlow, M.J. \& Clegg, R.E.S. 1995, MNRAS 272, 369

McLaughlin, M. \& Bell, K.L. 1993a, J. Phys. B: At. Mol. Opt. Phys. 26, 1797

McLaughlin, M. \& Bell, K.L. 1993b, J. Phys. B: At. Mol. Opt. Phys. 26, 3313

Nahar, S.N. 1995, ApJS 101, 423

Nahar, S.N. \& Pradhan, A.K. 1994, Phys. Rev. A 49, 1816

Oliva, E., Pasquali, A. \& Reconditi, M. 1996, A\&A 305, L21

Peng, J.F. \& Pradhan, A.K. 1995, ApJS 112, 151

Péquignot, D. \& Baluteau, J.-P. 1994, A\&A 283593

Ramsbottom, C.A., Bell, K.L. \& Stafford, R.P. 1996, ADNDT 63, 57

Sawey, P.M.J., Berrington, K.A., Burke, P.G. \& Kingston, A.E. 1990, J. Phys. B: At. Mol. Opt. Phys. 23, 4321

Sawey, P.M.J. \& Berrington, K.A. 1993, ADNDT 55, 81

Schöning. T. 1995, A\&A 299, L25

Schöning. T. \& Butler, K. 1995, A\&A 296, L29

Sigut, T.A. \& Pradhan, A.K. 1995, J. Phys. B: At. Mol. Opt. Phys. 28, 4879

Smits, D.P. 1991, MNRAS 248, 193

Smits, D.P. 1996, MNRAS 278, 683

Storey, P.J. 1994, A\&A 282, 999

Storey, P.J. \& Hummer, D.G. 1995, MNRAS 272, 41 